\title{
Classes of graphs with restricted interval models
}

\author{
Andrzej Proskurowski ${ }^{1}$ and Jan Arne Telle ${ }^{2}$ \\ ${ }^{1}$ University of Oregon, Eugene, Oregon, USA \\ ${ }^{2}$ University of Bergen, Bergen, Norway
}

received June 30, 1997, revised Feb 18, 1999, accepted Apr 20, 1999.

\begin{abstract}
We introduce $q$-proper interval graphs as interval graphs with interval models in which no interval is properly contained in more than $q$ other intervals, and also provide a forbidden induced subgraph characterization of this class of graphs. We initiate a graph-theoretic study of subgraphs of $q$-proper interval graphs with maximum clique size $k+1$ and give an equivalent characterization of these graphs by restricted path-decomposition. By allowing the parameter $q$ to vary from 0 to $k$, we obtain a nested hierarchy of graph families, from graphs of bandwidth at most $k$ to graphs of pathwidth at most $k$. Allowing both parameters to vary, we have an infinite lattice of graph classes ordered by containment.
\end{abstract}

Keywords: interval graphs, pathwidth, bandwidth

\section{Introduction}

Interval graphs model the intersection structure of a set of intervals of any linearly ordered structure, and have applications in fields as diverse as VLSI channel routing, molecular biology and scheduling. Our interest in subgraphs of interval graphs stems in part from the completion problem [5] and also from the elusiveness of results relating several graph parameters based on linear layouts of graphs. Pathwidth is a graph parameter closely associated with interval graphs, of importance to both algorithmic and structural graph theory, and with applications to VLSI layout $[6,11]$. Bandwidth is another widely studied graph parameter, with applications to sparse matrices [1], and notorious for the difficulty of its computation even for trees [3]. Recently, in a study of problems motivated by molecular biology, Kaplan and Shamir [5] showed a somewhat surprising connection between bandwidth and pathwidth, using the well-known notion of proper interval graphs, or equivalently unit interval graphs. A similar connection was shown by [2] based on a variant of the pursuit-evasion game of Cops and Robbers, where the Cop searches a graph by visiting its vertices in an attempt to apprehend the Robber that moves with a finite speed along graph edges. Depending on the rules of the game, the minimum speed needed by the Robber to avoid capture reflects either the bandwidth, or the pathwidth of the graph. In this paper we generalize and extend this connection. We fill in the gap between bandwidth and pathwidth, by giving a class of graphs parameterized by two integers where varying one parameter changes bandwidth gradually into pathwidth. 
In the next section we introduce $q$-proper interval graphs and give some preliminary definitions and results related to these graphs. In section 3, we give a forbidden induced subgraph characterization for $q$-proper interval graphs. In section 4 , we fix a maximum clique size, $k+1$, and vary $q$ from 0 to $k$ to study subgraphs of $q$-proper interval graphs with maximum clique size $k+1$. We characterize these nested families of graphs, that range from bandwidth $k$ to pathwidth $k$ graphs as $q$ ranges from 0 to $k$, by properties of their restricted path-decompositions. By varying both $q$ and $k$ we obtain an infinite lattice of graph classes, ordered by containment. In section 5 we investigate structural properties of these graphs by studying the edge maximal graphs and their minimal separators. In section 6 we show that these classes provide alternative characterizations of $k$-caterpillars and $k$-paths, and we introduce the class of $k$-rays as a compact and simple characterization of graphs of bandwidth at most $k$.

\section{$2 q$-proper interval graphs}

We use standard graph theory notation, as can be found, for instance, in [4]. An interval model of a graph can be specified by the left and right endpoints for each interval; these can be expressed as integers $l(v)$ and $r(v)$, respectively. The interval graph with this interval model has a vertex for each interval and an edge $u v$ if and only if the (closed) intervals $[l(v), r(v)]$ and $[l(u), r(u)]$ have non-empty intersection.

Definition 2.1 A graph $G$ is a q-proper interval graph if it has an interval model such that no interval is properly contained in more than $q$ other intervals.

Fact 2.2 0-proper interval graphs are usually called proper interval graphs.

In general, an interval $\left[a_{1}, b_{1}\right]$ is said to be properly contained in interval $\left[a_{2}, b_{2}\right]$ if either $a_{1}>a_{2}$ and $b_{1} \leq b_{2}$ or $a_{1} \geq a_{2}$ and $b_{1}<b_{2}$. We show that in the context of avoiding proper containments in interval models it suffices to deal only with proper containments where strict inequalities hold on both sides.

Definition 2.3 Interval $\left[a_{1}, b_{1}\right]$ is 2-sided properly contained in interval $\left[a_{2}, b_{2}\right]$ if $a_{1}>a_{2}$ and $b_{1}<b_{2}$.

Theorem 2.4 For any interval model $M$ of an interval graph $G$ we can find a new interval model $N$ of $G$ such that the only proper interval containments of $N$ are the 2-sided proper interval containments of $M$.

Proof. Without loss of generality (w.l.o.g.), let all interval endpoints of $M$ be integers between 1 and some maximum value $\max$. For each vertex $v$, define $\epsilon(v)=1 /(r(v)-l(v)+2)$ We construct a new interval model $N$ of $G$ by replacing the interval $[l(v), r(v)]$ of every vertex $v$ by $[l(v)-\epsilon(v), r(v)+\epsilon(v)]$. Note that no new proper containments or nonempty intersections of intervals are introduced by these changes. Additionaly, all proper interval containments which were not 2-sided in $M$ are not proper in $N$.

The following corollary is immediate.

Corollary 2.5 If an interval graph $G$ is not q-proper interval then in any interval model of $G$ we can find an interval which is 2-sided properly contained in at least $q+1$ other intervals.

\section{Forbidden subgraphs}

A graph $G$ is said to be $H$-free, for a fixed graph $H$, if it does not contain an induced subgraph isomorphic to $H$. We denote by $K_{q}$ the $q$-clique ( $q$ completely connected vertices) and by $K_{p, q}$ the complete bipartite graph (with partitions of $p$ and $q$ vertices). 
Definition 3.1 We denote by $T_{q}$ the graph consisting of a $K_{q}$ and 3 additional non-adjacent vertices, each adjacent to every vertex of the $K_{q}$.

In the following, we generalize the earlier characterization of proper interval graphs to the class of $q$-proper interval graphs.

Theorem 3.2 [10] $G$ is a 0-proper interval graph if and only if $G$ is a $T_{1}$-free (equivalently, $K_{1,3}$-free) interval graph.

Theorem 3.3 $G$ is a q-proper interval graph if and only if $G$ is a $T_{q+1}$-free interval graph.

Proof. (only if) Assume $G$ contains an induced $T_{q+1}$, consisting of a copy of $K_{q+1}$ and the 3 non-adjacent vertices $a, b, c$, and consider an interval model of $G$. Let (w.l.o.g.) the interval of $b$ lie entirely between intervals of $a$ and $c$ and let $I$ be the (non-empty) common intersection of all the intervals corresponding to vertices of $K_{q+1}$ intersect. Since intervals of $a, b, c$ must each intersect $I$, the interval of $b$ must be properly contained in $I$ and thereby $G$ is not $q$-proper interval.

(if) Assume that $G$ is interval but not $q$-proper and take any integer interval model of $G$. Modify this model by fixing the overall minimum left and maximum right endpoints and in turn for each vertex of $G$ extending its interval left and right as far as possible, with integer endpoints only, while maintaining an interval model of $G$. Let $b$ be a vertex whose interval is 2-sided properly contained in all intervals of some vertex set $K$, with $|K| \geq q+1$, as guaranteed by Corollary 2.5. Let the interval of $x \in K$ have the leftmost right endpoint $r(x)$ (of vertices in $K$ ). By our construction, if we extend the interval of $b$ to $r(x)$, the resulting interval model does not represent the graph $G$. Hence $b$ acquired a new neighbor $c \notin K$ such that $r(b)<l(c) \leq r(x)$. This means that $c$ is (in $G$ ) adjacent to every vertex in $K$ but not to $b$. Similarly we cannot move the left endpoint of $b$ to the left because of some vertex $a$ with similar properties as $c$. Note that $a$ and $c$ are not neighbors since the interval of $b$ lies entirely between their intervals. Thus $G$ contains an induced $T_{q+1}$.

\section{An infinite lattice of graph classes}

In this section we define an infinite lattice of graph classes, ordered by containment, which will serve to elucidate the relationship between graphs of bandwidth at most $k$ and graphs of pathwidth at most $k$. We first define bandwidth and pathwidth.

Definition 4.1 $G$ has bandwidth at most $k$ if there exists a bijection $f: V(G) \rightarrow\{1,2, \ldots,|V(G)|\}$ such that for every edge uv of $G$ we have $|f(u)-f(v)| \leq k$.

Definition 4.2 [11] A path-decomposition of a graph $G$ is a sequence of bags, subsets of the vertex set $V(G)$ of $G$, whose union is $V(G)$, such that for each edge of $G$ there is a bag containing both its end-vertices and the bags containing any given vertex are consecutive in the sequence. The maximum cardinality of a bag, minus one, is called the width of the path-decomposition. $G$ has pathwidth at most $k$ if and only if it has a width $k$ path-decomposition.

The pathwidth parameter can also be defined in terms of restricted interval graphs.

Definition 4.3 Given a path-decomposition $X_{1}, \ldots, X_{m}$ of a graph $G$, the interval model corresponding to it is given by $l(v)=\min \left\{i: v \in X_{i}\right\}$ and $r(v)=\max \left\{i: v \in X_{i}\right\}$. We say the path-decomposition specifies an embedding of $G$ into the interval graph with this interval model. 
Theorem 4.4 [6] $G$ is the subgraph of an interval graph with maximum clique size at most $k+1$ if and only if $G$ has pathwidth at most $k$.

In a recent paper, Kaplan and Shamir [5] show a connection between bandwidth and interval graphs.

Theorem 4.5 [5] $G$ is the subgraph of a 0-proper interval graph with maximum clique size at most $k+1$ if and only if $G$ has bandwidth at most $k$.

A definition analogous to the one above, for graphs of pathwidth at most $k$, follows immediately from Theorem 4.4 using the notion of $q$-proper interval graphs:

" $G$ is the subgraph of a $k$-proper interval graph with maximum clique size at most $k+1$ if and only if $G$ has pathwidth at most $k . "$

We fill in the gap between these two definitions of bandwidth and pathwidth, by defining a class of graphs parameterized by two integers $k$ and $q$ where varying the parameter $q$ from 0 to $k$ gradually moves the class from graphs of bandwidth at most $k$ to graphs of pathwidth at most $k$.

Definition 4.6 We denote by $B_{k, q}$, for integers $0 \leq q \leq k$, the class of graphs that are subgraphs of $q$-proper interval graphs with maximum clique size at most $k+1$.

Fact $4.7 B_{k, k}$ and $B_{k, 0}$ contain precisely the graphs of pathwidth at most $k$ and bandwidth at most $k$, respectively.

This fact follows from Theorems 4.4 and 4.5. Trivially, we have that $B_{k, q} \subseteq B_{k^{\prime}, q^{\prime}}$ for any $k \leq k^{\prime}$ and $q \leq q^{\prime}$. Hence, the graph classes $\left\{B_{k, q}: 0 \leq q \leq k\right\}$, ordered under containment, form an infinite lattice, where the least upper bound of $B_{k, q}$ and $B_{k^{\prime}, q^{\prime}}$ is $B_{\max }\left\{k, k^{\prime}\right\}, \max \left\{q, q^{\prime}\right\}$ and their greatest lower bound is $B_{\min }\left\{k, k^{\prime}\right\}, \min \left\{q, q^{\prime}\right\}$.

We conclude this section by giving an alternative characterization of the graphs in $B_{k, q}$ in terms of a restricted path-decomposition.

Definition 4.8 $A$ full width $k$ path-decomposition $X_{1}, \ldots, X_{m}$ has all bags of cardinality $k+1$ and $\mid X_{i} \cap$ $X_{i+1} \mid=k$ for $1 \leq i<m$.

Analogous restrictions have previously been applied to the study of tree-decompositions of graphs. Note that in a full path-decomposition $X_{1}, \ldots, X_{m}$ each bag except $X_{1}$ is the leftmost (lowest-numbered) bag for exactly one vertex and similarly each bag except $X_{m}$ is the rightmost (highest-numbered) bag for exactly one vertex.

Theorem 4.9 $G \in B_{k, q}$ if and only if $G$ has a full width $k$ path-decomposition $X_{1}, \ldots X_{m}$ where each vertex that does not belong to either bag $X_{1}$ or $X_{m}$ appears in at least $k-q+1$ bags.

Proof. (if) Assume $G$ has a path-decomposition $X_{1}, \ldots, X_{m}$ as in the statement of the Theorem. Consider the corresponding interval model $l(v)=\min \left\{i: v \in X_{i}\right\}$ and $r(v)=\max \left\{i: v \in X_{i}\right\}$. If $G$ is not $q$-proper we find, by Corollary 2.5, an interval 2 -sided properly contained in at least $q+1$ other intervals. We therefore find $k-q+1$ consecutive bags, $X_{i}, X_{i+1}, \ldots, X_{i+k-q}$, with $i>1$ and $i+k-q<m$ that share $q+2$ vertices. Each of the bags $X_{i+1}, \ldots, X_{i+k-q}$ has to be the leftmost bag for at least one of the remaining vertices in bag $X_{i+k-q}$. But this is a contradiction, since for these $k-q$ bags there are only $k-q-1$ such vertices. We conclude that $G$ is $q$-proper. 
(only if) Consider a $q$-proper integer interval model of a supergraph of $G$ with maximum clique size $k+1$. W.1.o.g. we can assume that no two intervals are equal. We show that this supergraph of $G$, and therefore $G$ itself, has the desired path-decomposition. Order vertices of $G$ by left endpoint of intervals $u_{1}, u_{2}, \ldots, u_{n}$, thus $u_{i} \in V(G)$ with $l\left(u_{i}\right) \leq l\left(u_{i+1}\right)$ and by right endpoints of intervals, $v_{1}, v_{2}, \ldots v_{n}$, breaking ties arbitrarily. We construct a full width $k$ path-decomposition of $G$ defined by $X_{1}=\left\{u_{1}, u_{2}, \ldots, u_{k+1}\right\}$ and $X_{i}=X_{i-1} \cup\left\{u_{k+i}\right\} \backslash\left\{v_{i-1}\right\}$ for $2 \leq i \leq n-k$. Note that $v_{1} \in X_{1}$ (and similarly $v_{i-1} \in X_{i-1}, 2 \leq i \leq n-k$ ) as otherwise the maximum clique size would exceed $k+1$. By construction, all bags thus have size $k+1$ and are distinct, and this is indeed a path-decomposition of $G$ since every pair of vertices corresponding to intersecting intervals belongs to a common bag. Let $v$ be a vertex not contained in bags $X_{1}$ or $X_{n-k}$ which appears in the least number of bags, $p$. There are $p$ vertices, including $v$, whose leftmost bag is among these bags. There are therefore $k-p+1$ vertices other than $v$ that are shared by all these $p$ bags, and each of these vertices belongs to more bags than $v$ does. The interval of $v$ must therefore have been properly contained in the interval of $k-p+1$ vertices. Since the interval model is $q$-proper we conclude that $k-p+1<q+1$ and therefore $p>k-q$ so that each vertex not in bags $X_{1}$ or $X_{n-k}$ belongs to at least $k-q+1$ bags.

\section{Edge maximal graphs}

In this section we investigate several important structural properties of graphs in $B_{k, q}$ by focusing on edge maximal graphs in these classes. Our first goal will be to show that the full path-decompositions of Theorem 4.9 specify an embedding into an edge-maximal graph. First some preliminary definitions and results.

Definition 5.1 We denote by $C_{k, q}$, for integers $0 \leq q \leq k$, the class of edge maximal graphs in $B_{k, q}$.

Note that $C_{k, q} \subseteq C_{k, q^{\prime}}$ for $q \leq q^{\prime}$ and $C_{k, q} \cap C_{k^{\prime}, q}=\emptyset$ for $k<k^{\prime}$.

For a connected graph $G$, a subset of vertices $S \subseteq V(G)$ is a separator of $G$ if there exist vertices $u$ and $v$, not in $S$, which are disconnected by removal of $S . S$ is a minimal separator of $G$ if no proper subset of $S$ is a separator.

Definition 5.2 For a graph $G$ we define the separator graph $S(G)$ to be the union of all subgraphs of $G$ induced by minimal separators of $G$.

Lemma 5.3 If $G \in C_{k, q}$ with full width $k$ path-decomposition $X_{1}, \ldots, X_{m}$ then the minimal separators of $G$ are $\left\{Y_{i}=X_{i} \cap X_{i+1}\right\}_{1 \leq i \leq m-1}$.

Proof. We show that $Y_{i}, 1 \leq i \leq m-1$ is a minimal separator. Let $u$ be a vertex for which $X_{i}$ is a rightmost bag and let $v$ be a vertex for which $X_{i+1}$ is a leftmost bag. Clearly, $Y_{i}$ is a separator since its removal disconnects $u$ and $v$. No proper subset of $Y_{i}$ is a separator since both $u$ and $v$ are adjacent to all vertices of $Y_{i}$ (otherwise $G$ would not be maximal), and the removal of $Y_{i}$ separates $G$ into two connected graphs containing $u$ and $v$, respectively. Moreover, any separator of $G$ must properly contain some $X_{i} \cap X_{i+1}$ in order to split its interval representation into two disjoint parts.

We can now show that the full path-decomposition of $G \in B_{k, q}$ of Theorem 4.9 specifies an embedding of $G$ into a graph in $C_{k, q}$. 


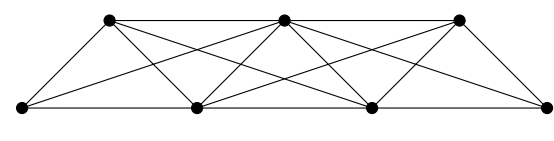

G
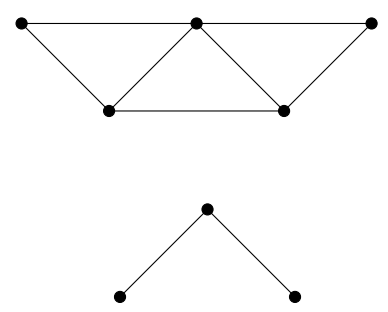

$S(S(G))$

Fig. 1: The 3-ray on 7 vertices (a graph in $C_{2,2}$ ), its separator graph the 2-ray on 5 vertices (a graph in $C_{2,1}$ ), and its separator graph the 1-ray on 3 vertices (a graph in $C_{2,0}$ ).

Lemma 5.4 $G \in C_{k, q}$ if and only if $G$ has an interval model corresponding to a full width $k$ pathdecomposition $X_{1}, \ldots, X_{m}$ with $k-q+1$ being the minimum number of bags to which any vertex not in $X_{1}$ or $X_{m}$ belongs.

Proof. The forward direction of the proof is a corollary to Theorem 4.9. For the other direction, we note that by Theorem $4.9 G$ is a subgraph of a graph in $C_{k, q}$. Moreover, intersections of adjacent bags have size $k$ and by Lemma 5.3 these are precisely the minimal separators of $G$. Any connected graph with no $(k+2)$-clique where all minimal separators induce $K_{k}$ is a $k$-tree [12]. The only subgraphs of graphs in $C_{k, q}$ that are $k$-trees are the maximal graphs and hence $G \in C_{k, q}$.

We turn to another main result of this section. Proskurowski [7] shows that for $k>0$ the separator graph of a $k$-caterpillar (a graph in $C_{k, k}$ ), is a $\left(k-1\right.$ )-caterpillar (a graph in $C_{k-1, k-1}$ ). The following Theorem generalizes this result (see Figure 1 for an example.)

Theorem 5.5 Let $q$ and $k$ be integers such that $0 \leq q \leq k$ and $k>0$. We then have

$$
\left\{\mathcal{S}(G): G \in C_{k, q}\right\}= \begin{cases}C_{k-1, q} & \text { if } q<k \\ C_{k-1, k-1} & \text { if } q=k\end{cases}
$$

Proof. We first show that for $G \in C_{k, q}$ where $q<k, S(G) \in C_{k-1, q}$. It follows by Lemma 5.3 that $\left\{Y_{i}=X_{i} \cap X_{i+1}\right\}_{1 \leq i \leq m-1}$ forms a path-decomposition of $S(G)$ with the corresponding interval model. Note that all bags in $\left\{Y_{i}\right\}$ are distinct, since the equality of $Y_{i}$ and $Y_{i+1}$ would imply that $Y_{i}=X_{i} \cap X_{i+2}$. Since all bags $X_{i}$ are distinct, this would mean that $X_{i+1}$ has a vertex appearing only in this bag, which contradicts Theorem 4.9. Each bag $Y_{i}, 1 \leq i<m$, has size $k$ by Lemma 5.4. Moreover, since each vertex of $G$ appears in at least $k-q+1$ bags of $\left\{X_{i}\right\}_{1 \leq i \leq m}$ it must appear in at least $k-q$ bags of $\left\{Y_{i}\right\}_{1 \leq i<m}$. Any bag $Y_{i}, 1 \leq i<m-1$ is the rightmost bag for exactly one vertex, since otherwise the corresponding $X_{i+1}$ would be the rightmost for two vertices in $G$. Similarly, each bag $Y_{i}, 1<i \leq m$ is leftmost for exactly one vertex. From Lemma 5.4 we therefore conclude that $S(G)$ is a graph in $C_{k-1, q}$.

In the above argument, the only place we assume $q<k$ is in the argument that all bags $Y_{i}, 1<i \leq m$ are distinct. In the case of $q=k$ we may have $Y_{i}=Y_{i+1}$. By removing all duplicate bags from 
$\left\{Y_{i}\right\}_{1 \leq i<m}$, we obtain a path-decomposition of $S(G)$ with otherwise the same properties as above. Since $k=q$ we need only show the trivial restriction that each vertex appears in at least one bag. This proves that $S(G)$ is in $C_{k-1, k-1}$.

To prove equality of the two graph classes, $\left\{S(G): G \in C_{k, q}\right\}=C_{k-1, q}$ for $0 \leq q \leq k$, it remains to show that for any graph $H$ in $C_{k-1, q}$ with a full width $k-1$ path-decomposition $Y_{1}, \ldots, Y_{m-1}$ there is a graph $G$ in $C_{k, q}$ with $S(G)$ isomorphic to $H$. Let us define the edge maximal graph $G$ whose interval model corresponds to the path-decomposition $X_{i}=Y_{i-1} \cup Y_{i}$ for $1<i<m$ and $X_{1}=Y_{1} \cup\{u\}$ and $X_{m}=Y_{m} \cup\{v\}$ with new vertices $u$ and $v$ not appearing in $H$. $G$ has $H$ as its separator graph and satisfies also the remaining criteria, as it is easy to check using Lemma 5.4. For $q=k-1$, we already showed that $G \in C_{k, k-1}$ and hence $G \in C_{k, k}$ with $S(G)=H$.

\section{Caterpillars, paths and rays}

In this section we continue the investigation of the edge-maximal graph classes $C_{k, q}$, providing alternative characterizations of $k$-caterpillars and $k$-paths, and introducing the class of $k$-rays as a compact and simple characterization of graphs of bandwidth at most $k$. We also give a simple algorithm finding, for a given $k$-caterpillar $G$, the minimum $q$ for which $G \in C_{k, q}$.

In the study of graphs with a tree structure or a linear structure, the $k$-trees, $k$-caterpillars and $k$-paths are well-known (note that some authors call $k$-caterpillars " $k$-paths", but we follow the earlier terminology introduced in [7, 13] which implies that 1-paths are exactly paths, just as 1-trees are exactly trees). Their iterative definitions are related as follows: (i) The complete graph on $k+1$ vertices is a $k$-tree. (ii) A $k$-tree with $n+1>k+1$ vertices is obtained from a $k$-tree on $n$ vertices by adding a new vertex adjacent to a $k$-clique, and iterative applications of this operation suffice to construct any $k$-tree. (iii) A vertex of degree $k$ in a $k$-tree is called a $k$-leaf. (iv) A $k$-path is a $k$-tree with two $k$-leaves. (v) A $k$-caterpillar is a $k$-tree consisting of a $k$-path with additional $k$-leaves ("hair" vertices) adjacent to some of its separator $k$-cliques.

It is well-known that $k$-trees are precisely the edge maximal graphs of treewidth $k$ and similarly $k$ caterpillars are precisely the edge maximal graphs of pathwidth $k$. Takahashi et al. [13] introduce the analogous graph parameter for $k$-paths by saying that a graph has proper pathwidth at most $k$ if and only if it is a subgraph of a $k$-path. (Note that Kaplan and Shamir in their paper [5] give the name "proper pathwidth" to a different parameter which they later show to be equivalent to bandwidth.) The following graph classes have the most rigid linear structure among generalizations of paths.

Definition 6.1 We denote by $k$-rays the class of edge-maximal graphs of bandwidth at most $k$.

See Figure 1 for an example of $k$-rays.

Theorem 6.2 $C_{k, k}, C_{k, k-1}$ and $C_{k, 0}$ are precisely the $k$-caterpillars, $k$-paths and $k$-rays, respectively.

Proof. The claims for $k$-caterpillars and $k$-rays follow from the above comments and Fact 4.7. To prove the claim for $k$-paths, we need to show that $k$-paths are precisely the graphs in $C_{k, k-1}$. By Theorem 4.9, we know that any graph $G$ in $C_{k, k-1}$ has a full width $k$ path-decomposition $X_{1}, \ldots, X_{m}$ where each vertex not in $X_{1}$ or $X_{m}$ belongs to at least 2 bags. By Lemma 5.4 we know that the graph with the corresponding interval model is a graph in $C_{k, k-2+1}=C_{k, k-1}$, hence $G$ itself has this interval model. Moreover, $C_{k, k-1} \subseteq C_{k, k}$ so that $G$ is a $k$-caterpillar and we need only show that $G$ has at most two vertices of degree $k$. By definition there are exactly two vertices appearing only in $X_{1}$ and $X_{m}$. Every 
other vertex in $G$ must appear in at least two disjoint bags $X_{i}$ and $X_{i+1}$, each containing $k$ other vertices, and must therefore have at least $k+1$ neighbors.

Corollary 6.3 For each positive integer $n$ and any $k<n, C_{k, 0}$ contains precisely one graph (up to isomorphism) with $n$ vertices $\left\{v_{1}, \ldots, v_{n}\right\}$. This graph has interval model $l\left(v_{i}\right)=i, r\left(v_{i}\right)=i+k$.

Proof. This follows from the definition of full width $k$ path-decompositions and Lemma 5.4 since with $q=0$ the only path-decomposition satisfying those restrictions, up to a re-labeling of vertices, is given by $\left\{X_{i}\right\}_{1 \leq i \leq n-k}$ where $X_{i}=\left\{v_{i}, v_{i+1}, \ldots, v_{i+k}\right\}$.

The following characterization of $k$-rays follows immediately from the above:

Corollary 6.4 Let $G$ be a graph. The following statements are equivalent:

1. G has bandwidth at most $k$.

2. $G \in B_{k, 0}$.

3. $G$ is a subgraph of the $k$-ray on $|V(G)|$ vertices.

4. $G$ has a path-decomposition $\left\{X_{i}\right\}_{1 \leq i \leq n-k}$ where $X_{i}=\left\{v_{i}, v_{i+1}, \ldots, v_{i+k}\right\}$ for some labeling of its vertices $V(G)=\left\{v_{1}, \ldots, v_{n}\right\}$.

We conclude this section with a simple algorithm for the following problem: Given a $k$-caterpillar $G$ what is the minimum value of $q$ for which $G \in C_{k, q}$ ?

Let a full path-decomposition of a $k$-caterpillar $G$ be $\mathcal{P}(G)=\left\{X_{i}\right\}_{0 \leq i \leq m}$, this is the unique width- $k$ path-decomposition in which no bag is a subset of another.

For a bag $X_{i}, 0<i \leq m$, of $\mathcal{P}(G)$, let us define the entry vertex $v_{i}$, to be the only vertex in $X_{i} \backslash X_{i-1}$. Let the exit vertex of $X_{i}, u_{i}$, be the only vertex in $X_{i} \backslash X_{i+1}$. We will say that $v_{i}$ replaces $u_{i}$. For the vertex $u_{0}$ appearing only in $X_{0}$, any other vertex in $X_{0}$ can be said to replace it. Similarly, the vertex $v_{m}$ appearing only in $X_{m}$ can be said to replace any other vertex in $X_{m}$.

The width-(k-1) path-decomposition of $H=\mathcal{S}(G)$ is given by bags $Y_{i}=X_{i} \cap X_{i+1}=X_{i+1} \backslash\left\{v_{i+1}\right\}$. We have shown in Theorem 5.5 that, for $q<k, G \in C_{k, q} \Rightarrow H \in C_{k-1, q}$. When $q<k-1, H$ is thus a $(k-1)$-path. We will now prove the inverse relation.

Lemma 6.5 Given a k-path $G$ and its separator graph $H=\mathcal{S}(G)$, for all $q<k, H \in C_{k-1, q} \Rightarrow G \in$ $C_{k, q}$.

Proof. Let us assume that $G$ has an induced subgraph isomorphic to $T_{q+1}$. We will show that so does $H$. Let the three mutually non-adjacent (independent) vertices of the copy of $T_{q+1}$ in $G$ be $w_{1}, w_{2}$ and $w_{3}$. Consider the path-decomposition $\mathcal{P}(G)=\left\{X_{i}\right\}_{0 \leq i \leq m}$ and wlog. let $w_{2}$ be the entry vertex of some $X_{i}, w_{1}$ be the exit vertex of some $X_{j}, j<i$ and $w_{3}$ be the entry vertex of some $X_{l}, i<l$. Since all the neighbors of $w_{2}$ share bags with $w_{2}$, we can always choose $w_{1}$ to be the exit vertex of $X_{i-1}$ and $w_{3}$ to be the entry vertex of the bag succeeding the bag of which $w_{2}$ is the exit vertex. Thus $w_{2}$ is in $X_{i} \cap \ldots \cap X_{l-1}$. Since $G$ is not a $k$-caterpillar, $w_{2}$ is in more than one bag and $i<l-1$. The copy $J$ of $K_{q+1}$ is in $X_{j} \cap \ldots \cap X_{l}$ and therefore also in the corresponding bags of a path decomposition of $H, Y_{i-1} \cap \ldots \cap Y_{l-1}$. The vertices $\operatorname{exit}\left(Y_{i-1}\right)$, entry $\left(Y_{i}\right)$ and entry $\left(Y_{l-1}\right)$ are independent in $H$, and thus, together with $J$ form in $H$ an induced subgraph isomorphic to $T_{q+1}$. 
Theorem 6.6 For a $k$-path $G$, the smallest value of $q$ for which $G \in C_{k, q}$ is the smallest $q$ for which $H=\mathcal{S}(G) \in C_{k-1, q}$.

The theorem follows from Lemma 6.5 and suggests the following high-level classification algorithm determining the smallest $q$ for which a given $k$-caterpillar $G$ is in $C_{k, q}$ :

Algorithm 6.7 :

Decomposition: Construct a path-decomposition $\mathcal{P}(G)=\left\{X_{i}\right\}_{0 \leq i \leq m}$. Set $q=k$.

Hair? If $X_{i} \cap X_{i-1}=X_{i} \cap X_{i+1}$ for some $i>0$, then $G \notin C_{k, k-1}$. Return $q$.

Iteration: While $G$ contains no hair vertices

Find $H=S(G)$.

Decrement q. Set $G=H$.

Return: $q-1$ i.e $G \in C_{k, q-1}$ and $G \notin C_{k, q-2}$.

A path-decomposition $\mathcal{P}(G)$ of $G$ can be constructed in linear time by finding vertices of degree $k$ and their neighborhoods in $G$, removing the $k$-leaves and iterating the process. Each construction of the separator graph $\mathcal{S}(G)$ and determination of the presence (or absence) of hair vertices can be done in linear time using, for instance, the string representation of $G$ (cf. [8]).

\section{Future Research}

We intend to investigate further algorithmic issues related to $q$-proper interval graphs. Indeed, in [9], we give some preliminary results related to the complexity of computing various graph parameters associated with the graph classes $\left\{B_{k, q}: 0 \leq q \leq k\right\}$. These graph classes form an infinite lattice when ordered by containment. Various graph parameters can be defined by fixing a particular chain or a set of elements in the lattice and asking for the least class in the chain containing a given graph. Pathwidth and bandwidth are examples of such parameters defined by the diagonal $q=k$ and the line $q=0$ of the lattice, respectively.

\section{References}

[1] E. Cuthill and J. McKee, Reducing the bandwidth of sparse symmetric matrices, Proc. Nat. Conf. ACM 157-172, 1969.

[2] F.V. Fomin, Helicopter search problems, bandwidth and pathwidth, Discrete Applied Mathematics (85)1 (1998) pp. 59-70.

[3] M. Garey, R. Graham, D. Johnson and D. Knuth, Complexity results for bandwidth minimization, SIAM J. Applied Math, vol. 34. no. 3, May 1978.

[4] M.C. Golumbic, Algorithmic Graph Theory and Perfect Graphs, Academic Press, 1980.

[5] H. Kaplan and R. Shamir, Pathwidth, bandwidth and completion problems to proper interval graphs with small cliques, SIAM J.Computing 25 (3) 540-561 (1996). 
[6] R. Möhring, Graph problems related to gate matrix layout and PLA folding, in Computational Graph Theory, Computing Suppl. 7 pp.17-51, Springer-Verlag, 1990.

[7] A. Proskurowski, Separating graphs in $k$-trees: cables and caterpillars, Discrete Mathematics 49 (1984) 275-285.

[8] A. Proskurowski, F. Ruskey and M. Smith, Analysis of algorithms for listing equivalence classes of $k$-ary strings, SIAM J. Discr. Math. 11 (1), 94-109 (1998)

[9] A. Proskurowski and J.A. Telle, From bandwidth k to pathwidth k, Proceedings ICTCS'98 - 5th Italian Conference on Theoretical Computer Science, Prato, Italy, November 9-11 1998, World Scientific Publishing, pp. 90-101.

[10] F. Roberts, Indifference graphs, in Proof Techniques in Graph Theory, F.Harary, ed. pp. 139-146, Academic Press, New York, 1969.

[11] N. Robertson and P. Seymour, Graph Minors I. Excluding a forest, Journal of Combinatorial Theory Series B 35 (1983) 39-61.

[12] D. Rose, On simple characterization of $k$-trees, Discrete Mathematics 7 (1974), 317-322.

[13] A. Takahashi, S. Ueno and Y. Kajitani, Minimal acyclic forbidden minors for the family of graphs with bounded path-width, Discrete Mathematics vol.127 (1994) 